\title{
BMJNeurology Open Moyamoya vasculopathy in a young Caucasian woman with significant methamphetamine use
}

\author{
Tiffany Lin, ${ }^{1}$ Charmaine Yam, ${ }^{1,2}$ Su-Ling Lai, ${ }^{3}$ Geoffrey Cloud ${ }^{1,2}$
}

To cite: Lin T, Yam C, Lai $\mathrm{S}$-L, et al. Moyamoya vasculopathy in a young Caucasian woman with significant methamphetamine use. BMJ Neurology Open 2020;2:e000066. doi:10.1136/ bmjno-2020-000066

Accepted 10 May 2020

\section{Check for updates}

(C) Author(s) (or their employer(s)) 2020. Re-use permitted under CC BY-NC. No commercial re-use. See rights and permissions. Published by BMJ.

${ }^{1}$ Department of Neurology, Alfred Health, Melbourne, Victoria, Australia

${ }^{2}$ Department of Clinical Neuroscience, Monash University Central Clinical School, Melbourne, Victoria, Australia

${ }^{3}$ Department of Radiology, Alfred Health, Melbourne, Victoria, Australia

Correspondence to

Dr Tiffany Lin;

tiffanylin71@gmail.com

\begin{abstract}
Background Moyamoya is a rare cerebrovascular disorder seen predominantly in Asian populations. Methamphetamine use is a recognised cause of stroke in young people, but its pathophysiology is not fully understood. The incidence of moyamoya vasculopathy in methamphetamine-associated stroke is unknown due to a lack of sufficient data. We present a rare case of moyamoya syndrome in a young Caucasian woman with methamphetamine-associated stroke.

Case A 31-year-old Caucasian woman presented with progressive right arm weakness, speech disturbance and seizures on a background of escalating methamphetamine use in the 9 months prior to admission. She did not have a personal or family history of stroke. MRI revealed both embolic and watershed infarcts in bilateral frontal regions and CT angiography showed development of new lenticulostriate collateral vessels. Digital subtraction angiography confirmed steno-occlusive disease of the bilateral anterior circulations and a 'puff of smoke' appearance.

Conclusion In young patients who present with stroke with unclear aetiology, it is important to obtain a thorough substance use history. Moyamoya vasculopathy should be considered when evaluating the pathophysiology of stroke in young people.
\end{abstract}

\section{BACKGROUND}

Moyamoya disease (MMD) is a rare cerebrovascular disease characterised by stenosis of bilateral internal carotid arteries and development of dilated collateral vessels. ${ }^{1}$ Incidence varies across ethnic populations, with highest prevalence in Japan. ${ }^{12}$ Its pathogenesis is poorly understood and is likely multifactorial. It is associated with certain conditions, such as haematological disorders, infection and autoimmune diseases. ${ }^{1-3}$ Mutations in RNF213 on chromosome 17q25.3 have been linked to an increased risk of developing MMD. ${ }^{3}$ However, this mutation shows marked frequency variations across populations and has not been detected in non-Asian patients. ${ }^{3}$ Other susceptibility genes have not been validated in independent studies, and overall, genetic associations are not strong enough to explain the pathology of MMD. ${ }^{3}$ We present a rare case of moyamoya vasculopathy in a young Caucasian woman associated with significant methamphetamine use.

\section{CASE PRESENTATION}

A 31-year-old Caucasian woman presented with 3 months of progressive right arm weakness, speech disturbance and seizures. Neurological examination showed dysarthria, dysphasia (reduced verbal fluency and word finding difficulties), right-sided hemiparesis and hemisensory loss. There were no signs of systemic disease, intercurrent illness, stigmata of neurofibromatosis or Down syndrome. She had no personal or family history of stroke. She was an active smoker of 10 cigarettes a day for 10 years. She was also a chronic cannabis and methamphetamine user, which escalated in the 9 months prior to admission to daily methamphetamine smoking.

Brain MRI revealed both bilateral embolic and watershed infarcts in frontal regions. CT angiography showed development of new lenticulostriate collateral vessels, suggestive of moyamoya pathology (figure 1A). Digital subtraction angiography (DSA) demonstrated steno-occlusive disease of bilateral anterior circulations, confirming the diagnosis (figure 1B).

Transthoracic echocardiography did not show a cardioembolic source of stroke. Lumbar puncture, vasculitis screen, autoimmune screen and thyroid function studies were unremarkable. Hepatitis and viral serology were negative. She was not hypertensive.

A repeat MRI demonstrated a new asymptomatic left frontal lobe infarct, which may have been related to the DSA procedure or embolism from her stenotic disease (figure 1C-E). She was subsequently started on clopidogrel in addition to aspirin to prevent further intracranial artery-to-artery embolism. Nimodipine was prescribed to 

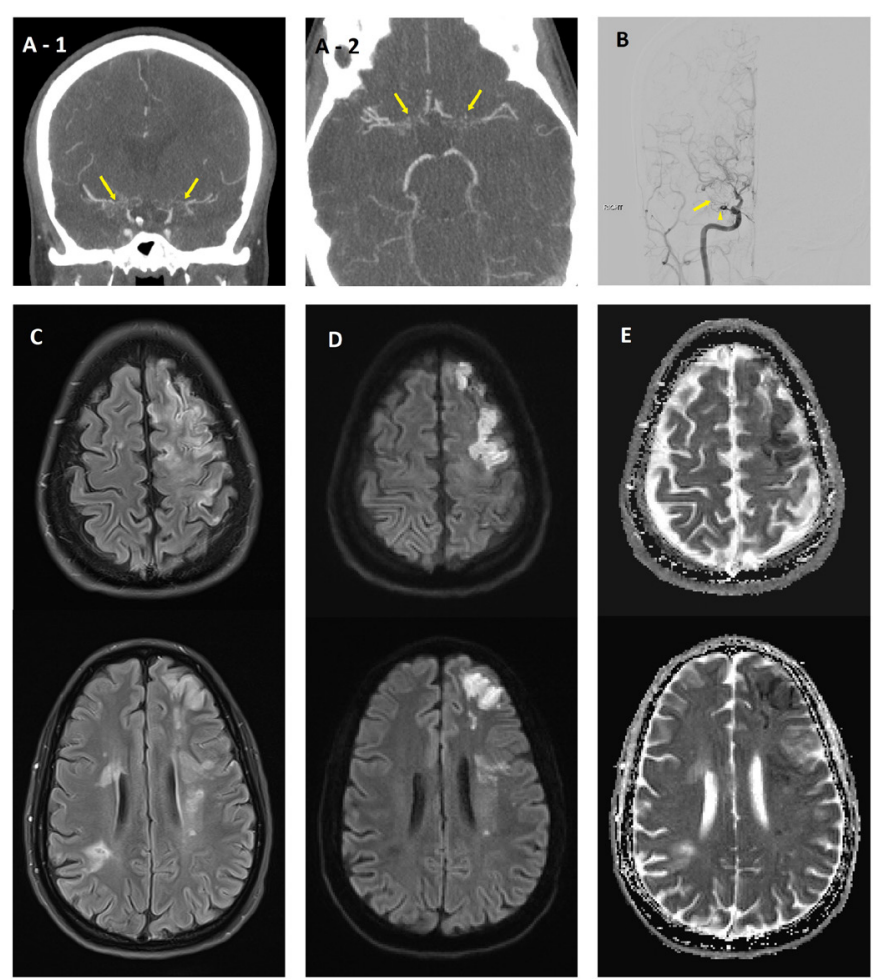

Figure 1 Representative imaging findings of the clinical course. (A) Coronal (1) and axial (2) CT angiogram demonstrating development of abnormal lenticulostriate vessels in bilateral internal carotid arteries. (B) DSA demonstrating steno-occlusive disease of the right anterior circulation with 'puff of smoke' appearance. (C-E) MRI with fluid attenuation inversion recovery $(C)$, diffusion-weighted (D) and apparent diffusion coefficient (E) sequences demonstrating multiple foci of acute infarction within the left frontal lobe as well as chronic established infarcts within the right corona radiata and periventricular white matter. DSA, digital subtraction angiography.

treat any underlying cerebral vasospasm and carbamazepine was started for seizure prophylaxis.

She underwent an 8-week period of inpatient rehabilitation. At outpatient review 4 months later, she had clinically improved and experienced no further stroke symptoms while on dual antiplatelet therapy and ceasing methamphetamine. Repeat MRI showed no new ischaemic changes but the appearance of the intracranial vessels was unchanged. She continues to be managed conservatively with consideration of neurosurgery in the event of further neurological deterioration.

\section{DISCUSSION AND CONCLUSION}

MMD is a progressive steno-occlusive disease of bilateral internal carotid arteries, causing development of dilated collateral vessels and a 'puff-of-smoke' angiographic appearance. ${ }^{4}$ First reported in 1957 in Japan, the term moyamoya was coined to reflect these characteristic angiographic findings. ${ }^{4}$ Moyamoya syndrome terminology ${ }^{\mathrm{i}} \mathrm{s}$ used when moyamoya vessels are found in those with associated conditions such as autoimmune diseases, infection diseases and haematological disorders, whereas MMD refers to those without these conditions. ${ }^{124}$

Moyamoya has a bimodal distribution, with childhood onset around 5 years of age and adult onset around 40 years of age. ${ }^{1}$ The clinical picture varies from an asymptomatic benign course to disabling ischaemic or haemorrhagic stroke. ${ }^{1}$ Children usually present with ischaemic events whereas adults more commonly present with intracerebral haemorrhage. ${ }^{1}$ In Japan, MMD affects almost twice as many females as males. ${ }^{5}$

Revascularisation surgery is the most effective treatment for haemorrhagic MMD but randomised controlled trials are lacking to support this option for ischaemic MMD. ${ }^{6}$ Revascularisation aims to restore blood supply, stabilise cerebrovascular haemodynamics and prevent further bleeding. ${ }^{6}$ Conservative management with antiplatelet therapy is usually recommended, although its effect on course of disease is unproven. ${ }^{6}$

Our case has several rare features. Moyamoya is most common in East Asian populations and is extremely rare among Caucasians (0.94/100 000 in Japan, 0.41/100 000 in China and 0.09/100 000 in North America). ${ }^{2}$ Our patient was Caucasian and presented with ischaemic events when in adults intracerebral haemorrhage is the far more common presentation. Furthermore, extensive workup to assess for presence of moyamoya-associated medical conditions did not reveal any of these to be present. In particular, there was no evidence of autoimmune disease, which has been associated with moyamoya in both East Asian and Caucasian populations. ${ }^{7-9}$

A 2015 review by Hever et al found that that MMD in Caucasians differs to those in Asians by a markedly lower prevalence and lower rates of haemorrhage in adults, later time of onset and a more benign course of disease. ${ }^{10}$ They propose a theory of a distinct Western phenotype in MMD, which could possibly be due to a difference in underlying pathophysiology. ${ }^{10}$

This patient's chronic methamphetamine use is notable. Methamphetamine use is a recognised cause of stroke in young people and accounts for significant worldwide morbidity. ${ }^{11}$ An Australian nation-wide study found that one in five fatal strokes among those aged 15-44 involved psychostimulants, of which methamphetamine was the most common substance implicated. ${ }^{12}$ However, incidence of moyamoya vasculopathy in patients with psychostimulant use is unknown due to a lack of sufficient data. ${ }^{11}$ To our knowledge, only one case has ever been reported of moyamoya vasculopathy being found in a patient presenting with poly-drug overdose. ${ }^{13}$ Toxicology testing found these drugs to be alcohol, cocaine, 3,4-me thylenedioxy-methamphetamine and 2C-I, but not those of the amphetamine class. ${ }^{13}$

The pathophysiology of methamphetamineassociated stroke is uncertain. ${ }^{10}$ A review of all fatal methamphetamine-associated strokes in Australia from 2009 to 2015 suggested that drug-induced hypertensive events increased the likelihood of vessel wall damage and development of cerebral vasculitis. ${ }^{14}$ Other studies 
propose that direct vascular toxicity, hypertension, vasospasm or atherosclerosis may be the underlying mechanism. ${ }^{11} 15$

We present a case of previously undescribed moyamoya vasculopathy in a young Caucasian woman with methamphetamine-associated stroke. The mechanism is not understood, and it is unclear whether moyamoya vessel development was secondary to or preceding significant methamphetamine use in this patient but we suspect the former.

As methamphetamine use increases worldwide, ${ }^{11}$ this case emphasises the importance of taking a thorough substance use history when evaluating young patients who present with stroke and consider moyamoya vasculopathy as an underlying disease process.

Contributors All authors were involved in the clinical care of this patient. The initial draft of the manuscript was prepared by TL, which was edited by CY and GC. Radiological evaluation and figure 1 was provided by S-LL. All authors approved the final draft.

Funding The authors have not declared a specific grant for this research from any funding agency in the public, commercial or not-for-profit sectors.

Competing interests None declared.

Patient consent for publication Obtained.

Provenance and peer review Not commissioned; internally peer reviewed.

Data availability statement Data sharing is not applicable as no datasets generated and/or analysed for this study.

Open access This is an open access article distributed in accordance with the Creative Commons Attribution Non Commercial (CC BY-NC 4.0) license, which permits others to distribute, remix, adapt, build upon this work non-commercially, and license their derivative works on different terms, provided the original work is properly cited, appropriate credit is given, any changes made indicated, and the use is non-commercial. See: http://creativecommons.org/licenses/by-nc/4.0/.

\section{REFERENCES}

1 Scott RM, Smith ER. Moyamoya disease and moyamoya syndrome. N Engl J Med 2009;360:1226-37.

2 Kuroda S, Houkin K. Moyamoya disease: current concepts and future perspectives. Lancet Neurol 2008;7:1056-66.

3 Duan L, Wei L, Tian Y, et al. Novel susceptibility loci for moyamoya disease revealed by a genome-wide association study. Stroke 2018;49:11-18.

4 Fukui M. Guidelines for the diagnosis and treatment of spontaneous occlusion of the circle of Willis ('moyamoya' disease). Research Committee on spontaneous occlusion of the circle of Willis (moyamoya disease) of the Ministry of health and welfare, Japan. Clin Neurol Neurosurg 1997;99 Suppl 2:S238-40.

5 Kuriyama S, Kusaka Y, Fujimura M, et al. Prevalence and clinicoepidemiological features of moyamoya disease in Japan: findings from a nationwide epidemiological survey. Stroke 2008;39:42-7.

6 Acker G, Fekonja L, Vajkoczy P. Surgical management of moyamoya disease. Stroke 2018;49:476-82.

7 Bower RS, Mallory GW, Nwojo M, et al. Moyamoya disease in a primarily white, midwestern US population: increased prevalence of autoimmune disease. Stroke 2013;44:1997-9.

$8 \mathrm{Kim} \mathrm{SJ}$, Heo KG, Shin HY, et al. Association of thyroid autoantibodies with moyamoya-type cerebrovascular disease: a prospective study. Stroke 2010:41:173-6.

9 Sigdel TK, Shoemaker LD, Chen R, et al. Immune response profiling identifies autoantibodies specific to moyamoya patients. Orphanet $J$ Rare Dis 2013;8:45.

10 Hever P, Alamri A, Tolias C. Moyamoya angiopathy - Is there a Western phenotype? Br J Neurosurg 2015;29:765-71.

11 Lappin JM, Darke S, Farrell M. Stroke and methamphetamine use in young adults: a review. J Neurol Neurosurg Psychiatry 2017;88:1079-91.

12 Darke S, Duflou J, Kaye S, et al. Psychostimulant use and fatal stroke in young adults. J Forensic Sci 2019;64:1421-6.

13 Drees JC, Stone JA, Wu AHB. Morbidity involving the hallucinogenic designer amines MDA and 2C-I. J Forensic Sci 2009;54:1485-7.

14 Darke S, Lappin J, Kaye S, et al. Clinical characteristics of fatal Methamphetamine-related stroke: a national study. J Forensic Sci 2018;63:735-9.

15 Ho EL, Josephson SA, Lee HS, et al. Cerebrovascular complications of methamphetamine abuse. Neurocrit Care 2009;10:295-305. 